\title{
Tuberculose pleural após uso de adalimumabe na doença de Crohn: relato de caso
}

\author{
Pleural tuberculosis after using adalimumab in Crohn's disease: a case report
}

\author{
RODRIGO ROCHA BATISTA ${ }^{1}$, PAULA SALVADOR DE TOLEDO ${ }^{2}$, IDBLAN CARVALHO DE ALBUQUERQUE 3 , \\ GALDINO JOSÉ SITONIO FORMIGA ${ }^{4}$
}

${ }^{I}$ Filiado da Sociedade Brasileira de Coloproctologia; Médico Residente do Serviço de Coloproctologia do Hospital Heliópolis - São Paulo (SP), Brasil. ${ }^{2}$ Médica Residente de Cirurgia Geral do Hospital Heliópolis - São Paulo (SP), Brasil.

${ }^{3}$ Titular da Sociedade Brasileira de Coloproctologia; Médico Assistente do Serviço de Coloproctologia do Hospital

Heliópolis - São Paulo (SP), Brasil. ${ }^{4}$ Titular da Sociedade Brasileira de Coloproctologia; Chefe do Serviço de

Coloproctologia do Hospital Heliópolis - São Paulo (SP), Brasil.

BATISTA RR, TOLEDO PSD, ALBUQUERQUE IC, FORMIGA GJS. Tuberculose pleural após uso de adalimumabe na doença de Crohn: relato de caso. Rev bras Coloproct, 2011;31(1): 85-88.

RESUMO: $O$ tratamento da doença de Crohn perineal é feito pela combinação da terapia medicamentosa e da cirúrgica. A terapia biológica é importante devido à eficácia clínica na indução e manutenção de remissão da doença. No entanto, devido à ação imunomoduladora e imunossupressora, o uso de biológicos como infliximabe e adalimumabe eleva o risco de infecções oportunistas. Relatou-se o caso de paciente feminino, 28 anos, diagnosticada com doença de Crohn perineal, em uso de azatioprina e adalimumabe. Há sete dias com tosse seca, febre vespertina e dispneia. Ao exame físico, febril, desidratada, diminuição do murmúrio vesicular nos campos médio e inferior do hemitoráx direito e cicatriz de fistulotomia anorretal sem sinais flogísticos. A radiografia de tórax mostrou derrame pleural em hemitórax direito, e a análise do líquido pleural constatou adenosina deaminase elevada, nível de glicose normal e citologia diferencial com $\mathbf{8 8 \%}$ de monomorfonucleares. Foi estabelecido o diagnóstico de tuberculose pleural, e a paciente foi medicada com esquema tríplice (rifampicina, isoniazida e pirazinamida) por seis meses associada à prednisona $40 \mathrm{mg} / \mathrm{dia}$, por um mês, com posterior desmame do corticoide. Atualmente, encontra-se assintomática e em uso de ciprofloxina $1 \mathrm{~g} /$ dia para a doença de Crohn perineal.

Palavras-chave: doença de Crohn; terapia biológica; fator de necrose tumoral alfa; adalimumabe; tuberculose pleural.

\section{INTRODUÇÃO}

A Doença de Crohn (DC) é uma doença inflamatória intestinal, caracterizada por inflamação transmural que favorece a formação de abscessos e fístulas. A manifestação perineal da DC ocorre com frequência variável, sendo maior nos pacientes com doença colorretal ${ }^{1}$.

O tratamento da DC perineal (DCP) alcança melhores resultados ao associar terapia medicamentosa e cirúrgica. Dos medicamentos utilizados, destaca-se a terapia biológica devido à eficácia clínica na indução e manutenção de remissão da doença ${ }^{1-5}$. No entanto, ao neutralizar o fator de necrose tumoral alfa (TNF- $\alpha$ ), exercendo, por isso, ação imunomoduladora e imussupressora, o uso de biológicos como infliximabe e adalimumabe eleva o risco de infecções oportunistas ${ }^{1,2,6}$.

O objetivo deste trabalho é relatar um caso de tuberculose (TB) pleural secundária ao uso de adalimumabe para o tratamento da DCP complexa.

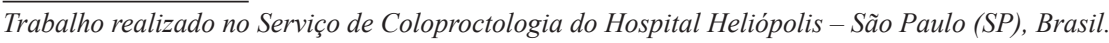

Recebido em: 24/06/2009

Aprovado em: 14/04/2011 


\section{RELATO DE CASO}

Paciente feminino, 28 anos, com diagnóstico de DCP complexa, há três meses em uso de azatioprina (AZA) e há seis semanas em uso de adalimumabe após pesquisa para TB negativa (Figura 1). Queixa de tosse seca, febre vespertina e dispneia há sete dias. Antecedente cirúrgico de drenagem de abscesso isquiorretal com colocação de sedenho. Ao exame físico, paciente em regular estado geral, descorada, febril, desidratada, com diminuição do murmúrio vesicular nos campos médio e inferior do hemitoráx direito, estertores crepitantes e macicez à percussão. Ao exame proctológico, à inspeção, presença de cicatriz de fistulotomia anterolateral direita sem sinais flogísticos; ao toque retal, sem abaulamentos ou secreções.

Realizou-se radiografia de tórax (Figura 2), que mostrou derrame pleural em hemitórax direito até terço médio, e hemograma com hemoglobina de 10,9 g/ $\mathrm{dL}$ e leucograma com 4.830 células $/ \mathrm{mm}^{3}$ sem desvio. A paciente foi submetida à toracocentese diagnóstica, cuja análise do líquido pleural (LP) (Tabela 1) constatou adenosina deaminase elevada (ADA: 95,3 U/L), nível de glicose normal e citologia diferencial com $88 \%$ de monomorfonucleares.

Em função do exame clínico e dos exames complementares, estabeleceu-se o diagnóstico de TB pleural. Foi medicada com esquema tríplice (rifampicina, isoniazida e pirazinamida) por seis meses associada à prednisona $40 \mathrm{mg} / \mathrm{dia}$, por um mês,

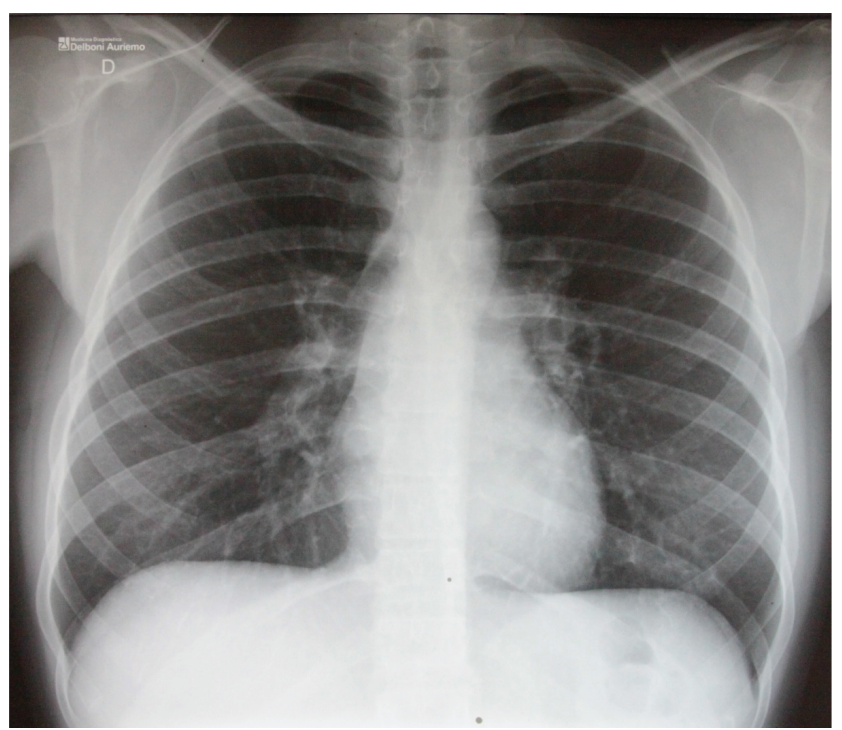

Figura 1. Radiografia de tórax normal.

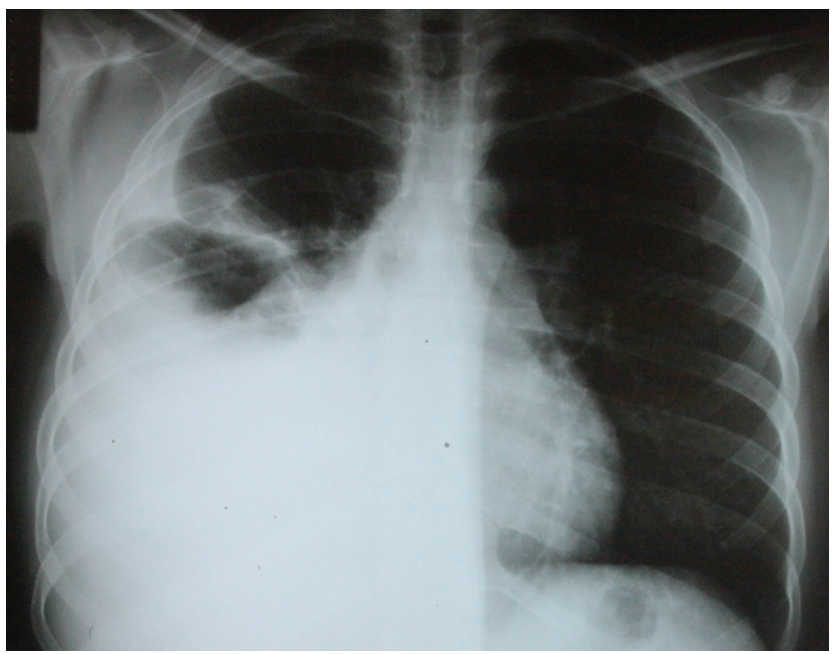

Figura 2. Radiografia de tórax com derrame pleural.

Tabela 1. Análise do líquido pleural.

\begin{tabular}{lc}
\hline Parâmetros & Resultados \\
\hline Cor e aspecto & Amarelo e turvo \\
Proteínas totais & $5,5 \mathrm{~g} / \mathrm{dL}$ \\
Albumina & $3,2 \mathrm{~g} / \mathrm{dL}$ \\
Desidrogenase lática & $1.249 \mathrm{U} / \mathrm{L}$ \\
Glicose & $118 \mathrm{mg} / \mathrm{dL}$ \\
Adenosina deaminase & $95,3 \mathrm{U} / \mathrm{L}$ \\
(ADA) & \\
Citologia & 3.300 leucócitos $/ \mathrm{mm}^{3}$ \\
Baciloscopia & (88\% nononucleares) \\
\hline
\end{tabular}

com posterior desmame do corticoide. O exame de escarro e a cultura do LP foram negativos para bacilo álcool-ácido resistente (BAAR). Atualmente, encontra-se assintomática e em acompanhamento ambulatorial, em uso de ciprofloxacina $1 \mathrm{~g} /$ dia para o tratamento da DCP.

\section{DISCUSSÃO}

O TNF- $\alpha$ é uma citocina proinflamatória multifuncional, que se encontra em níveis elevados na DC ativa, e está relacionada à amplificação e manutenção da disfunção inflamatória ${ }^{1,2,7}$. O TNF- $\alpha$ também se associa com a patogênese de diversas moléstias infecciosas, entre elas a $\mathrm{TB}^{8-10}$.

Com o advento dos imunomoduladores, cujo principal representante é a AZA, e da terapia biológica, representada principalmente pelo infliximabe 
(anti-TNF $\alpha$ 75\% humano) e mais recentemente pelo adalimumabe (anti-TNF $\alpha$ 100\% humano), conseguiuse melhor controle das doenças imunomediadas, notadamente a $\mathrm{DC}^{1,4,7,8}$

Por conta do efeito imunossupressor, antes do início do uso de imunomoduladores ou de agentes biológicos, devem ser descartadas doenças infecciosas ativas ou latentes, tais como: viroses, micoses profundas e TB. Portanto, a exclusão da TB pulmonar latente é obrigatória antes do uso de bloqueadores do TNF- $\alpha^{6,11}$. Segundo o Consenso Europeu de prevenção, diagnóstico e manejo de infecções oportunistas em pacientes com doença inflamatória intestinal, a pesquisa da TB deve ser realizada por meio da história clínica e epidemiológica, do exame físico, da radiografia de tórax e do teste cutâneo da tuberculina (PPD) ${ }^{6}$.

No presente relato, a paciente realizou o PPD e a radiografia de tórax antes de iniciar a AZA e o adalimumabe, os quais foram não-reator e a radiografia, normal. Entretanto, houve uma surpresa diante da complicação infecciosa oportunista, a qual foi suspeitada e tratada como TB pleural baseado no perfil bioquímico e citológico do LP e na radiografia de tórax, apesar de a cultura do LP e exame de escarro, com pesquisa de BAAR, terem sido negativas.
O comprometimento pleural representa a forma mais frequente da TB extrapulmonar e pode ser uma manifestação tanto da forma primária da doença (primo-infecção), quanto da reativação de uma infecção latente pelo M. tuberculosis. Na forma aguda, pode simular uma pneumonia bacteriana com tosse nãoprodutiva, febre, dispneia e dor torácica ventilatóriadependente. O LP, segundo os critérios de Light, é caracteristicamente um exudato, a baciloscopia é inespecífica e a positividade da cultura do LP é em torno de 10 a 35\%. A dosagem da ADA no LP é de extrema importância no diagnóstico da TB pleural, visto que apresenta sensibilidade e especificidade de $95 \%$ quando se utiliza o valor de corte maior que $40 \mathrm{U} / \mathrm{L}^{12}$.

Existe grande preocupação acerca da validade diagnóstica do PPD para diagnóstico de infecção latente pelo vírus $M$. tuberculosis, devido a sua baixa especificidade em indivíduos vacinados com BCG e baixa sensibilidade em pacientes com doenças inflamatórias imunomediadas. Para tentar melhorar a acurácia dos testes cutâneos, novas técnicas vêm sendo testadas ainda em caráter experimental ${ }^{6,11,13-15}$.

Assim, é necessário o acompanhamento rigoroso dos pacientes em uso de imunomoduladores e da terapia biológica para realizar o diagnóstico e tratamento precoce de infecções oportunistas.

\footnotetext{
ABSTRACT: The Crohn's disease perineal treatment is made by the combination of drug and surgery therapy. The biological therapy is important due to clinical efficacy in inducing and maintaining disease remission. However, because of immunomodulating and immunosuppressive effects, the use of biological as infliximab and adalimumab increases the risk of opportunistic infections. We report a case of a 28-year-old, female patient, diagnosed with Crohn's perineal disease, taking azathioprine and adalimumab, complaining of dry cough, evening fever, and dyspnea for seven days. On physical examination, febrile, dehydrated, decreased, breath sounds in the middle and lower fields of the right hemithorax and scar of anorectal fistulotomy without signs of inflammation. The chest radiograph showed pleural effusion in the right hemithorax, and the analysis of pleural fluid found high levels of adenosine deaminase, normal levels of glucose and cytology differential with $88 \%$ of mononuclear cells. With the diagnosis of pleural tuberculosis, the patient was treated with triple drug regimen (rifampicin, isoniazid, and pyrazinamide) for six months associated with prednisone $40 \mathrm{mg} / \mathrm{day}$, for one month, with subsequent weaning of corticosteroid. Currently, she is asymptomatic and in treatment with ciprofloxacin $1 \mathrm{~g}$ daily for perineal Crohn's disease.
}

Keywords: Crohn disease; biological therapy; tumor necrosis factor-Alpha; adalimumab; tuberculosis, pleural.

\section{REFERÊNCIAS}

1. Kotze PG, Albuquerque IC, Moraes AC, Vieira A, Souza F. Análise de custo-minimização entre o infliximabe (IFX) e o adalimumabe (ADA) no tratamento da doença de Crohn (DC). Rev Bras ColoProct. 2009;29(2):158-68.

2. Costamagna P, Furst K, Tully K, Landis J, Moser K, Quach L, et al. Tuberculosis associated with blocking agents against tumor necrosis factor-alpha. MMWR. 2004;53:683-6.

3. Aberra FN, Stettler N, Brensinger C, Lichtenstein GR, Lewis JD. Risk for active tuberculosis in patients with inflammatory bowel disease. Clinical Gastroenterol Hepatology . 2007;5(9):1070-5

4. Travis SPL, Stange EF, Le'mann M, Öresland TO, Chowers Y, Forbes A, et al. European evidence based consensus on 
the diagnosis and management of Crohn's disease: current management. Gut. 2006;55(Suppl I):16-35.

5. Panés J, Gomollón F, Taxonera C, Hinojosa J, Clofent J, Nos P. Crohn's disease: a review of current treatment with a focus on biologics. Drugs. 2007;67(17):2511-37.

6. Colombel JF, Sandborn WJ, Panaccione R, Robinson AM, Lau W, Li J, et al. Adalimumab safety in global clinical trials of patients with Crohn's disease. Inflamm Bowel Dis. 2009;15(9):1308-19.

7. Lichtenstein GR, Hanauer SB, Sandborn WJ; Practice Parameters Committee of American College of Gastroenterology. Management of Crohn's disease in adults. Am J Gastroenterol. 2009;104(2):465-83.

8. Rahier JF, Ben-Horin S, Chowers Y, Conlon C, De Munter P, D'Haens G, et al. European evidence-based Consensus on the prevention, diagnosis and management of opportunistic infections in inflammatory bowel disease. J Crohns Colitis. 2009;3(2):47-91

9. Lertxundi U, Mayo J, García M, Ruiz B, Aguirre C. Miliary tuberculosis following treatment with adalimumab. Farm Hosp. 2005;29(5):344-6.

10. Antolín J, Azahara M, Hernández C, Blanco M, Mao L, Cigüenza R. Tuberculous peritonitis after treatment with adalimumab. Scand J Infect Dis. 2008;40(8):677-8.
11. Lange C, Hellmich B, Ernst M, Ehlers S. Rapid immunodiagnosis of tuberculosis in a woman receiving antiTNF therapy. Nat Clin Pract Rheumatol. 2007;3(9):528-34.

12. Seiscento M, Conde MB, Dalcolmo MMP. Tuberculose pleural. J Bras Pneumol. 2006;32(4):174-81.

13. Lalvani A, Millington KA. Screening for tuberculosis infection prior to initiation of anti-TNF therapy. Autoimmun Rev. 2008;8(2):147-52.

14. Gomez-Reino JJ, Carmona L, Descalzo MA. Risk of tuberculosis in patients treated with tumor necrosis factor antagonists due to incomplete prevention of reactivation of latent infection. Arth Care Res. 2007;57(5):756-61.

15. Laffitte E, Janssens JP, Roux-Lombard P, Thielen AM, Barde C, Marazza G, et al. Tuberculosis screening in patients with psoriasis before antitumour necrosis factor therapy: comparison of an interferon-gamma release assay vs. tuberculin skin test. Br J Dermatol. 2009;161(4):797-800.

\section{Endereço para correspondência:}

GALDINO JOSÉ SITONIO FORMIGA

Serviço de Coloproctologia do Hospital Heliópolis

Rua Cônego Xavier, 276 - Nova Heliópolis

CEP 04231-030 - São Paulo (SP), Brasil

E-mail: gformiga@ig.com.br 